\title{
Evaluation of Antihyperglycemic Effect of Epilobium parviflorum in High Fat Diet Fructose Streptozotocin Induced Type 2 Diabetic Albino Wistar Rats
}

\author{
S. K. RANAJIT' ${ }^{1, *}$, K. N. KILLARI ${ }^{2}$, E. K. KILARI ${ }^{2}$ AND P. K. SAHU ${ }^{1}$
}

School of Pharmacy, Centurion University of Technology and Management, Odisha 767001, ${ }^{1}$ Heritage Vision Educational Trust, Bhubaneswar, Odisha, ${ }^{2}$ Andhra University College of Pharmaceutical Sciences, Visakhapatnam, Andhra Pradesh, India

\section{Ranajit et al.: Evaluation of Antihyperglycemic Effect of Epilobium parviflorum}

\begin{abstract}
The proposed work deals with pharmacological evaluation of aqueous leaf extract of Epilobium parviflorum for antihyperglycemic and antihyperlipidemic activity in high fat diet fructose streptozotocin induced type 2 Diabetes mellitus in albino wistar rats. The experimental protocol was designed on a total of 30 rats, equally divided into 5 groups (6 rats in each group). The high fat diet fructose was administered to all the groups except the normal control group throughout a $6 \mathrm{w}$ study period. A low dose of streptozotocin (35 $\mathrm{mg} / \mathrm{kg}$ body weight) is freshly prepared and administered intraperitoneally once after the initial $2 \mathrm{w}$ of high fat diet fructose administration. The animals were observed for $1 \mathrm{w}$ to induce diabetes. The two doses of aqueous leaf extract of Epilobium parviflorum $(200 \& 400 \mathrm{mg} / \mathrm{kg})$ and pioglitazone $(10 \mathrm{mg} / \mathrm{kg})$ were administered orally once daily throughout the rest $3 \mathrm{w}$. Various serum parameters including lipid profile, liver profile, renal profile and inflammatory biomarkers were estimated on $0^{\text {th }}$ and $6^{\text {th }} \mathrm{w}^{\text {of }}$ the study. All the individual test results are falling within the normal distribution of the test groups (treatment with leaf extract) when compared with disease control group with least variance $(\mathbf{p}<0.01)$. However, when compared to the standard group (pioglitazone treated), the results show too much variance. Increase in insulin release and decreased fasting blood sugar results are purely dose dependant. The histopathological studies of hepatocyte, nephron, adipocytes and islet of pancreatic beta cells were also exhibited excellent antihyperglycemic and antihyperlipidemic activity while the test group maintained normal cell architectures. With the assumption on the traditional curative assurance and scientific evidences, it may be concluded that Epilobium parviflorum contributes to the antihyperglycemic as well as antihyperlipidemic activity by maintaining the normal structure and function of hepatocytes, renal, pancreas and adipose function.
\end{abstract}

Key words: Epilobium parviflorum, fructose, streptozotocin, diabetes

The two most common epidemic metabolic disorders like diabetes mellitus and obesity are associated with hyperglycemia and hyperlipidemia respectively ${ }^{[1]}$. Very often these two types of metabolic disorders found in the same individual due to various reasons. The aberrant metabolism of carbohydrates, lipids and proteins are mainly the consequences of insulin dysregulation in the body ${ }^{[2]}$. The chronic downturn release of insulin and escalation of insulin resistance can increase the glucose level and produce severe morbidity and mortality. The associated comorbidities of diabetes are deterioration in visual sight, renal excretion, nervine senses, foot amputation and cardiovascular disease ${ }^{[3]}$.

Type 2 diabetes mellitus is going to abrupt in 400 million people worldwide by the y $2030^{[4]}$. The

*Address for correspondence

E-mail: sanrancol@gmail.com

May-June 2021 conventional therapeutic approaches for diabetes like subcutaneous insulin injection \& oral hypoglycemic drugs are well adapted by the patients still due to their inconvenience and severe side effects the strategy of therapeutic approaches is rejuvenated from time to time $^{[5]}$. Most of the researchers are searching for the therapeutic molecule from the herbal origin due to their easy availability, minimal cost and few side effects as compared to English medicine ${ }^{[6]}$.

This is an open access article distributed under the terms of the Creative Commons Attribution-NonCommercial-ShareAlike 3.0 License, which allows others to remix, tweak, and build upon the work non-commercially, as long as the author is credited and the new creations are licensed under the identical terms

Accepted 06 June 2021

Revised 05 February 2021

Received 16 July 2020

Indian J Pharm Sci 2021;83(3):547-555 
The Epilobium parviflorum is a small herb and is well known as willow herb. The genus Epilobium contains a variety of active principles like polyphenol and flavonol aglycones (ex. quercetin, kaempferol and myricetin $)^{[7]}$. There are 200 species of the same genus are distributed worldwide $^{[8]}$. It has tremendous therapeutic uses in the treatment of various diseases like prostate hyperplasia ${ }^{[9]}$, pain and inflammation by impaired the action of both the cyclooxygenase 1 and 2 enzymes, suppressed the growth of Escherichia coli and exerted antioxidant activity ${ }^{[10]}$. Various infections due to microbes, fungal and viral ${ }^{[8]}$. It has good antioxidant properties and has a high rate of free radicals scavenging activity than conventional antioxidants like Trolox and ascorbic acid $^{[11]}$. Based on tremendous traditional uses but lack of scientific evidence, the present study was aimed to ensure the therapeutic value of E. parviflorum by evaluating pharmacological investigation as a potent antihyperglycemic and antihyperlipidemic agent in high fat diet fructose streptozotocin (HFD-FRU-STZ) induced diabetic rat model.

\section{MATERIALS AND METHODS}

\section{Chemicals and drugs:}

The dried powder of aqueous leaf extract of Epilobium parviflorum, bearing batch No. NC/EPE/14001 is obtained from Green chem herbal extracts \& formulation, Bangalore. All the chemicals used in the study were labelled as an analytical grade. The streptozotocin (STZ), casein, fructose and other chemicals are procured from the Sisco Research Laboratories (SRL) Pvt. Ltd., Mumbai. The normal pellet diet is obtained from VRK Nutritional Solutions, Bangalore. The egg, coconut oil and suet (goat fat) are obtained the form from Visakhapatnam local market.

\section{Animals used:}

The male albino Wistar rats of age 3 mo, body weight about 180-250 g were procured from Albino research Lab., Hyderabad, A.P, India. The animals were well housed under standard well maintained 12:12 dark and light cycle in the standard environment temperature $\left(25 \pm 2^{\circ}\right)$ with relative humidity $50 \pm 10 \%$. The animals were free to access to water and ad libitum with a standard rodent diet. The present study was approved by the institutional animal ethical committee (IAEC) bearing the committee for the purpose of control and supervision of experiments on animals (CPCSEA) Registration No-516/PO/c/01/IAEC.

\section{Preparation of HFD:}

A modified HFD is prepared by slight alteration in the composition of the diet mentioned by Bigoniya $\mathrm{P}$ et al. $2012^{[12]}$. The composition includes $40 \%$ NPD (normal powder diet) $25 \%$ Suet (goat fat), $6 \%$ coconut oil, $10 \%$ fructose and $6 \%$ casein protein, $12 \%$ Egg protein. $0.5 \%$ of multivitamin and $0.5 \%$ of sodium chloride. Throughout the proposed study, the HFD is prepared freshly in the morning daily and given to the animals.

\section{Induction of diabetes mellitus:}

The 30 albino Wistar rats were separated into five groups consisting 6 animals each. The rats were fed with HFD-FRU throughout the study period of $6 \mathrm{w}$. After the completion of $2 \mathrm{w}$, the animals were subjected to overnight fasting followed by injection of a low dose of freshly prepared STZ $(35 \mathrm{mg} / \mathrm{kg})$ in $0.1 \mathrm{M}$ sodium citrate buffer ( $\mathrm{pH} 4.5)$ intraperitoneally ${ }^{[12]}$. All the animals are observed for the induction of phenotype diabetic model within $1 \mathrm{w}$ and fasting blood sugar (FBS) was estimated for confirmation. The animals in which FBS showed between 250 to $350 \mathrm{mg} / \mathrm{dL}$ are included in the study.

\section{Experimental protocol:}

In the present investigation, the 30 animals are divided into 5 groups with different treatment protocol such as, Group I (Normal control): all the animals were fed with normal diet; Group II (Diseased control): all the animals received HFD-FRU-STZ and distilled water as vehicle; Group III (Standard treatment): all the animals received HFD-FRU-STZ and treatment with pioglitazone (10mg/kg body weight); Group VI (Treatment): all the animals received HFD-FRUSTZ and treatment with E. parviflorum leaf extract $200 \mathrm{mg} / \mathrm{kg}$; Group V (Treatment): all the animals received HFD-FRU-STZ and treatment with E. parviflorum leaf extract $400 \mathrm{mg} / \mathrm{kg}$.

\section{Sample collection:}

The blood samples were collected from the retro-orbital plexus of overnight fasted rats. The fine capillary is inserted gently into the inner angle of the eye under light ether induced anesthetized rats. The blood is subjected to centrifuge at $4000 \mathrm{rpm}$ for $10 \mathrm{~min}$ to obtain serum.

\section{Estimation of physical parameters:}

The Physical parameters like body weight is determined on $0^{\text {th }}, 3^{\text {rd }}$ and $6^{\text {th }} \mathrm{w}$, whereas the food intake and water intake were determined on $1^{\text {st }}, 3^{\text {rd }}$ and $6^{\text {th }} \mathrm{W}$ of 
investigation by the help of digital weighing balance and measuring cylinder. A certain amount of food and water were given to the animals and the consumed amounts were determined by subtracting the remained amount from the initial amount over a period of $24 \mathrm{~h}$.

\section{Estimation of biochemical parameters:}

In the present investigation various parameters like FBS, glycosylated haemoglobin (HbAlc), liver profile [serum glutamic-oxaloacetic transaminase (SGOT), serum glutamic-pyruvic transaminase (SGPT) \& alkaline phosphatase (ALP)], lipid profile [triglyceride (TG), total cholesterol (TC), low density lipoprotein (LDL) and high density lipoprotein (HDL)], uric acid, creatinine are estimated by colorimetric method using Coral kits in semi autoanalyzer (evolution 3000), highsensitivity C-reactive protein (hs-CRP) was estimated by turbidimetry analysis using Excel kits in autoanalyzer (CAREX), tumor necrosis factor (TNF- $\alpha$ ) and insulin were estimated in Elisa microplate reader (Lisaquant 3000, TULIP Diagnostics Pvt Ltd). The homeostatic model assessment of insulin resistance (HOMA-IR) is calculated from the fasting insulin level and FBS level (Fasting insulin $\times \mathrm{FBS} / 22.5$ ) to assess the extent of insulin resistance by the following formula ${ }^{[13]}$. The oral glucose tolerance test (OGTT) is estimated $2 \mathrm{~d}$ before the completion of $6^{\text {th }} \mathrm{W}^{[14]}$.

\section{Histopathological study:}

The animals are sacrificed at the end of the $6^{\text {th }} \mathrm{w}$ and the vital organs like pancreas, kidney, and liver were isolated and kept in $10 \%$ neutral buffered formalin prior to histopathology. The organs are washed and embedded in paraffin and subjected to microtome. Three micron section were prepared and stained by haematoxylin and eosin dye. The stained sections were observed under phase contrast microscope (Labomed) (400x magnification).

\section{Statistical analysis:}

The results are expressed as Mean \pm SEM and the data are analyzed in Graph pad Prism 5 software. The data are analyzed by two way analysis of variance (ANOVA) followed by Bonferroni post-test. The significance values are indicated by $* * * p<0.001, * * p<0.01,{ }^{*} \mathrm{p}<0.05$ when compared to the diseased control group. The badge \# indicates insignificant results $(p>0.05)$ when compared to normal control group. Some parameters such as insulin, HOMA-IR, creatinine, uric acid, lipid profile and liver profile were statistically analysed using Dunnett's t-test.

\section{RESULTS AND DISCUSSION}

In the $3^{\text {rd }} \mathrm{w}$ of HFD-FRU modified diet administration of prior administration of STZ the body weight, food intake and water intake are increased compared to normal control group. The report on $6^{\text {th }} \mathrm{w}$ of successive treatment with various drugs showed the significant decrease in body weight and increase in both food intake and water intake in the diabetes control group, whereas treatment with pioglitazone and E. parviflorum decrease the food intake-water intake and increase the bodyweight when compared to the diabetes control group and are depicted in the Table 1 and fig. 1.

Table 2, represents the impart of treatment groups on serum FBS, HbA1c, insulin and HOMA-IR index. The administration of received HFD-FRU-STZ significantly increases the FBS, HbA1c and HOMA -IR index, despite significantly decrease the serum insulin level in the diabetic control group as compared to the normal control group. In contrast, all the over mentioned parameters of Pioglitazone and E. parviflorum treated groups are discriminated against to justify the antihyperglycemic activity in a dose-dependent manner (Table 2).

Fig. 2 represents the effect of various treated groups on OGTT. The serum sugar level of five groups is estimated at five different time points after the oral infusion of glucose. In the normal control rats, the blood glucose levels attained a high level at $60 \mathrm{~min}$ and fall down to a normal level after $180 \mathrm{~min}$. In the case of diabetic control in spite of fall down to its normal blood glucose level, it rose steadily. In the Pioglitazone \& E. parviflorum treated rats, the blood glucose levels

TABLE 1: ESTIMATION OF FOOD INTAKE AND WATER INTAKE IN DIFFERENT INTERVAL

\begin{tabular}{|c|c|c|c|c|c|c|}
\hline \multirow{2}{*}{ Group } & \multicolumn{3}{|c|}{ Food intake (gm) } & \multicolumn{3}{|c|}{ Water intake $(\mathrm{ml})$} \\
\hline & $1^{\text {st }} w$ & $3^{\text {rd }} w$ & $6^{\text {th }} w$ & $1^{\text {st }} w$ & $3^{\text {rd }} w$ & $6^{\text {th }} w$ \\
\hline Normal control & 17.67 & 19.67 & 20.33 & 69.33 & 70.33 & 71.12 \\
\hline Diseased control & 37.5 & 42.67 & 53.67 & 76.57 & 82.67 & 94.33 \\
\hline Standard drug & 37.28 & 41.33 & 45.9 & 76.33 & 80.59 & 85.66 \\
\hline E. parviflorum 200 & 38.33 & 43.33 & 51.17 & 74.33 & 78.96 & 88.63 \\
\hline E. parviflorum 400 & 36.83 & 42 & 48.33 & 75.33 & 80.67 & 87.45 \\
\hline
\end{tabular}


www.ijpsonline.com

TABLE 2: THE FBS, INSULIN, HOMA-IR AND HBA1c LEVEL ON $6^{\text {th }} \mathbf{W}$

\begin{tabular}{lcccl}
\hline Group & FBS $(\mathrm{mg} / \mathrm{dL})$ & INSULIN $(\mu \mathrm{Ul} / \mathrm{ml})$ & HOMA-IR (Mass units) & HbA1c $(\%)$ \\
\hline Normal control & $83.67 \pm 1.4$ & $18.36 \pm 0.86$ & $3.8 \pm 0.12$ & $4.65 \pm 0.19$ \\
Diseased control & $323.17 \pm 4.7^{\#}$ & $10.54 \pm 0.74^{\#}$ & $8.4 \pm 1.08^{\#}$ & $9.85 \pm 0.21^{\#}$ \\
Standard drug & $155.24 \pm 2.73^{* *}$ & $17.26 \pm 0.61^{* *}$ & $6.6 \pm 0.32^{*}$ & $6.91 \pm 0.09^{* *}$ \\
E. parviflorum 200 & $198.33 \pm 3.41^{*}$ & $12.68 \pm 0.88$ & $7.4 \pm 0.81$ & $7.73 \pm 0.69$ \\
E. parviflorum 400 & $183.46 \pm 3.18^{*}$ & $13.45 \pm 0.74^{*}$ & $7.0 \pm 0.27^{*}$ & $7.12 \pm 0.22^{*}$ \\
\hline
\end{tabular}

Note: The significance values are indicated by ${ }^{* *} \mathrm{p}<0.01,{ }^{*} \mathrm{p}<0.05$ when compared to the diseased control group. The badge \# indicates insignificant results $(p>0.05)$ when compared to normal control group

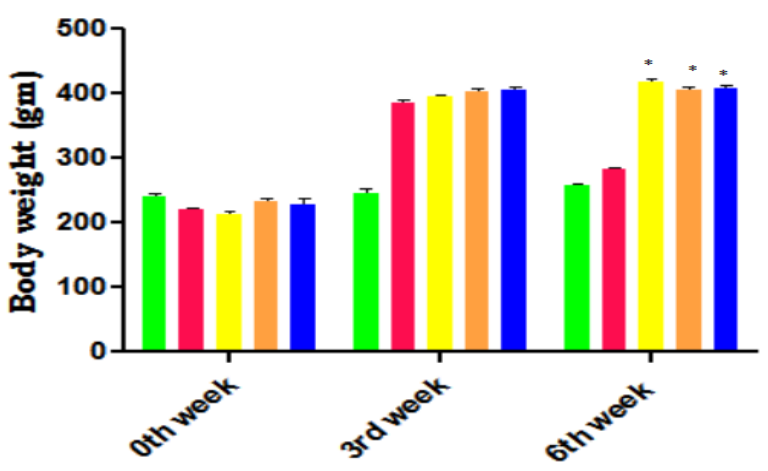

Fig. 1: Determination of body weight on different interval $(* \mathbf{p}<0.05)$ when compared to the diseased control group.

are falling down after $60 \mathrm{~min}$ and gradually reached its near about values of $0 \mathrm{~min}$ at $120 \mathrm{~min}$ (fig. 2). The E. parviflorum treated groups showed its potency in a dose-dependent manner.

The TNF- $\alpha$ and hs-CRP are estimated as they are involved in the pathogenesis of diabetes and obesity to produce insulin resistance. We found that, these two inflammatory biomarkers are significantly increased in the diabetic control group while compared to the normal groups. Treatment with pioglitazone and E. parviflorum has brought down their levels to near about its normal value. The E. parviflorum 400 has shown less potent than Pioglitazone treated group in reducing TNF- $\alpha$ and hs-CRP (Table 3).

Table 3 shows the effect of various treatment groups on hepatic enzymes like SGOT, SGPT and ALP. These hepatic biomarkers are significantly higher in the diabetic control group than the normal control group. Treatment with pioglitazone and E. parviflorum has prominently lowered these enzymes than the diabetic control group. Here also E. parviflorum 400 treated groups significantly reduced the hepatic enzymes than the diseased control group. In this regard, the pioglitazone has a more suppressive effect on these enzymes than E. parviflorum treated group (Table 3).

The increased level of creatinine and uric acid are two well-recognized parameters to define the diseased condition of the kidney. The diseased control rats had

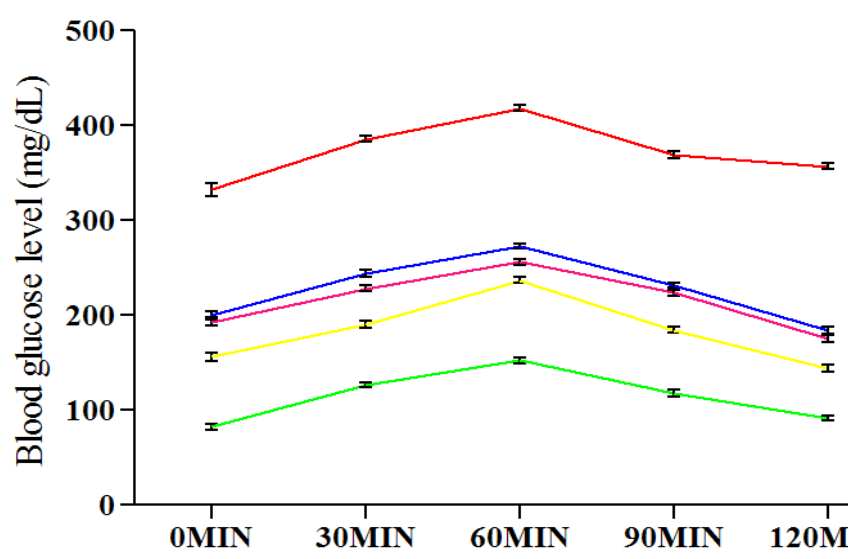

Fig. 2: Determination of oral glucose tolerance test

Note: The significance values are indicated by $* * \mathbf{p}<0.01$, ${ }^{*} \mathbf{p}<0.05$ when compared to the diseased control group. The badge \# indicates insignificant results $(p>0.05)$ when compared to normal control group.

increased serum creatinine and uric acid levels compared to normal control groups whereas the pioglitazone and E. parviflorum treated groups significantly reduced these renal biomarkers prominently when compared to the diseased control group (Table 3).

Impart of treatment on lipid profile (TG, TC, VLDL, HDL and LDL) the effect of various groups on lipid profiles is presented in Table 3. The lipid biomarkers like TG, TC, VLDL and LDL are significantly higher whereas HDL is found to be lower in the diabetic control group due to received HFD-FRU-STZ modified diet. Treatment with Pioglitazone and E. parviflorum had significant lowering action on serum TG, TC, VLDL and LDL level and amplified the HDL level to a significant level compared to the diseased control group (Table 3).

The histologic determination of various organs (fig. 3) like pancreas (A-E), liver (F-J), kidney (K-O) and adipose tissue (P-T) were done at the end of study. The photomicrographic representation of kidney, liver, pancreas and adipose tissue in the diabetic control group shows disorientation in the histological architecture by atrophy, shrinkage, necrosis of glomerulus \& renal tubules in case of the kidney; steatosis, hypertrophy of hepatic cells in case of the liver; decrease in the islet 
www.ijpsonline.com

TABLE 3: THE ESTIMATION OF VARIOUS PARAMETERS AT THE END OF 6th W

\begin{tabular}{|c|c|c|c|c|c|}
\hline Parameters & Normal control & Diseased control & Standard drug & E. parviflorum $200 E$. & - parviflorum 400 \\
\hline TNF a $(\mathrm{pg} / \mathrm{mL})$ & $12.17 \pm 1.17$ & $35.33 \pm 1.98$ & $19.67 \pm 1.45^{*}$ & $28.00 \pm 1.59^{* *}$ & $26.83 \pm 1.78^{*}$ \\
\hline hs $\operatorname{CRP}(\mathrm{mg} / \mathrm{dL})$ & $2.11 \pm 0.08$ & $6.40 \pm 0.11$ & $2.70 \pm 0.12^{*}$ & $3.87 \pm 0.14^{*}$ & $3.72 \pm 0.13^{*}$ \\
\hline SGOT (IU/dL) & $49.00 \pm 0.45$ & $116.50 \pm 7.21$ & $71.83 \pm 5.14^{* *}$ & $84.56 \pm 2.61^{*}$ & $81.33 \pm 5.45^{*}$ \\
\hline SGPT (IU/dL) & $18.83 \pm 1.11$ & $63.17 \pm 3.06$ & $46.83 \pm 2.83^{*}$ & $57.50 \pm 1.67^{*}$ & $53.67 \pm 2.39^{*}$ \\
\hline ALP(IU/L) & $112.83 \pm 1.72$ & $320.83 \pm 4.53$ & $176.50 \pm 5.29^{* *}$ & $194.50 \pm 2.20^{*}$ & $182.83 \pm 2.20^{*}$ \\
\hline Uric acid (mg/dL) & $3.5 \pm 0.15$ & $8.48 \pm 0.14$ & $4.9 \pm 0.17^{* *}$ & $6.92 \pm 0.14^{*}$ & $6.38 \pm 0.12^{*}$ \\
\hline Creatinine (mg/dL) & $0.5 \pm 0.01$ & $1.92 \pm 0.02$ & $0.75 \pm 0.03^{* *}$ & $1.1 \pm 0.02^{*}$ & $0.98 \pm 0.03^{*}$ \\
\hline $\mathrm{TG}(\mathrm{mg} / \mathrm{dL})$ & $104.33 \pm 2.80$ & $364.33 \pm 9.69$ & $134.17 \pm 2.66^{* *}$ & $203.33 \pm 3.34^{*}$ & $200.17 \pm 3.77^{*}$ \\
\hline $\mathrm{TC}(\mathrm{mg} / \mathrm{dL})$ & $85.17 \pm 2.34$ & $220.83 \pm 3.23$ & $120.33 \pm 2.89^{* *}$ & $147.33 \pm 3.31^{*}$ & $144.00 \pm 2.32^{*}$ \\
\hline $\operatorname{VLDL}(\mathrm{mg} / \mathrm{dL})$ & $20.87 \pm 0.56$ & $72.87 \pm 1.94$ & $26.83 \pm 0.53^{* *}$ & $40.67 \pm 0.67^{*}$ & $40.03 \pm 0.75^{*}$ \\
\hline $\mathrm{LDL}(\mathrm{mg} / \mathrm{dL})$ & $30.63 \pm 2.87$ & $132.13 \pm 2.66$ & $67.50 \pm 2.99^{* *}$ & $84.50 \pm 3.83^{*}$ & $83.13 \pm 2.93^{*}$ \\
\hline $\mathrm{HDL}(\mathrm{mg} / \mathrm{dL})$ & $33.83 \pm 0.98$ & $15.83 \pm 0.60$ & $26.00 \pm 1.06^{* *}$ & $22.17 \pm 0.79^{*}$ & $22.83 \pm 0.75^{*}$ \\
\hline
\end{tabular}

Note: The significance values are indicated by ${ }^{* *} \mathrm{p}<0.01,{ }^{*} \mathrm{p}<0.05$ when compared to the diseased control group. The badge \# indicates insignificant results $(p>0.05)$ when compared to normal control group
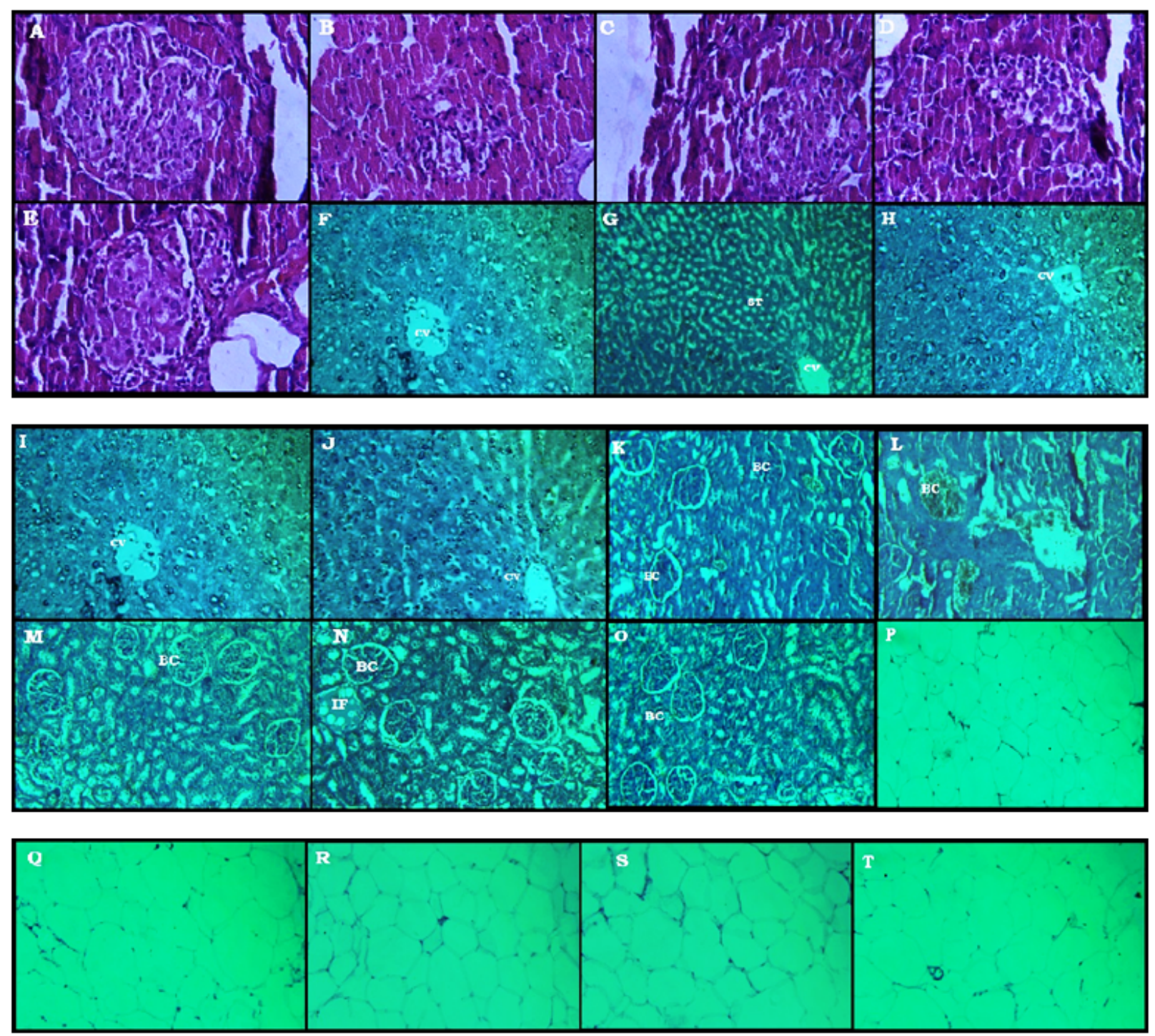

Fig. 3: The histopathological studies of various organs

Note: It represents the all five group's photomicrographs of Pancreas (A-E); Liver (F-J); Kidney (K-O); Adipose tissue (P-T) respectively, BC- Bowmen capsule; IF-Infiltration; CV- central vein. 
numbers of Langerhans, abnormal boundaries lining of the islet in case of the pancreas and enlarged size of adipocyte with increased deposition of fat content in case of adipose tissues are found. The tissue architectures of Pioglitazone and E. parviflorum treated groups are maintained and minimal changes are found when compared to the diseased control groups (fig. 3).

In the present investigation, the received HFD-FRUSTZ is used in the development of phenotype type 2 diabetes mellitus in albino Wistar rats. No doubt that a number of genetically manipulated animals like zucker rat, ob/ob mouse, OLEFT, etc ${ }^{[1]}$ models are being used in the study but those are not well adapted due to certain circumstances like cost factor, availability, breeding and maintenance. Therefore the researchers are searching for a new model to induce type 2 diabetes associate with hyperlipidemia. Hence in the present investigation, received HFD-FRU-STZ is used in order to induce the phenotype metabolic disorder in concern to abnormal carbohydrate and lipid metabolism as a similar report provided by other researchers ${ }^{[15,16]}$. Indeed the modification in the diet like consuming excess fat can directly increase the triglyceride (TG) and cholesterol levels in the diabetic conditions. As a consequence, there will be a deposition of fat in the abdominal as well in the dorsal part. This condition can lead to insulin resistant in peripheral tissues ${ }^{[15]}$.

Enriched fructose diet can impair the glucose absorption and accelerate the serum TG and cholesterol and it mimics the various symptoms of Type 2 diabetes-like increased insulin resistance, hyperlipidemia and renal diseases with raised blood pressure ${ }^{[16]}$. Earlier a number of researches are made on fructose-induced type 2 diabetes mellitus in animals ${ }^{[17-19]}$. The fructose mainly depended on the liver for its metabolism. It produces nonalcoholic fatty liver disease (NAFLD) by increasing hepatic denovo lipogenesis (DNL) and decreasing fatty acid beta $(\beta)$ oxidation. The various hepatic enzymes like fructokinase, aldolase (ALDOA) and tyrosine kinase are involved in the conversion of fructose into Acetyl co A. Thereafter the Acetyl co A is shifted to synthesize triglyceride by hepatic denovo lipogenesis. The hyper triglyceride level mostly produces hyperlipidemia and led to insulin resistance. In addition to this fructose also reduce the peroxisome proliferator-activated receptor (PPAR) $\alpha$ transcription thereby decrease in mitochondrial fatty acid oxidation and increase insulin resistance through diacylglycerol (DAG) production and Protein Kinase $\mathrm{C} \varepsilon(\mathrm{PKC} \varepsilon)$ activation. The DNL is an insulin-independent process ${ }^{[20]}$. The high fructose diet can induce insulin resistance in the skeletal muscles via NAFLD through activation of Toll-like receptor 4(TLR 4) and nuclear factor $\mathrm{KB}(\mathrm{NF} \mathrm{\kappa B})$ in the gut ${ }^{[21]}$.

The low dose of STZ and nicotinamide ${ }^{[22]}$; Multiple lowdose STZ ${ }^{[23]}$; low dose STZ with high-fat diet ${ }^{[16]}$ and high fructose $\operatorname{diet}^{[18]}$ is used by various researchers to induce the pathological similarity as diabetes mellitus. In the present investigation, the $35 \mathrm{mg} / \mathrm{kg}$ body weight of STZ is injected once after the $3 \mathrm{w}$ of received HFDFRU-STZ administered animal to develop type 2 diabetes mellitus in albino Wistar rats.

The bodyweight of received HFD-FRU induced rats are increased in the entire group except the diseased control group. The decreased in body weight implies the muscles mass wasting in the form of increased utilization of muscle protein for gluconeogenesis in the liver to compensate glucose crisis in insulin resistance. Treatment with E. parviflorum 400 there is a substantial improvement in their body weight, which indicates the sign of increased insulin sensitivity by preventing loss of body weight of diabetic rats (fig. 1). As a consequence of this there is a decrease in food intake and water intake in E. parviflorum 400 treated groups (Table 1). This decreased polyphagia and polydipsia are also indicated the therapeutic effect of $E$. parviflorum in HFD-FRU-STZ induced diabetic rats.

From the ancient researches the FBS level, HbAlc, serum insulin and OGTT are the most relevant parameters to define diabetes mellitus. The liver upregulates the glucose synthesis in the body by means of gluconeogenesis and glycogenolysis through glycolytic, tricarboxylic acid (TCA) cycle, glycogenic and hexose monophosphate (HMP) pathways during diabetes mellitus ${ }^{[24]}$. The severity of hyperglycemia can alleviate the non-enzymatic protein glycation process, where the free amino acids from body proteins covalently bind with the glucose and produce various comorbidities. The blood haemoglobin follows the glycosylation process in hyperglycemic state and converts to $\mathrm{HbA} 1 \mathrm{c}$. The endocrine pancreas consists of $70 \%$ of $\beta$ cells in islet cells that release insulin. The main role of insulin is to maintain the body's glucose levels via increasing their utilization in vital organs like liver, fat and skeletal muscles.

The HOMA-IR is calculated for all the groups to form serum FBS levels and insulin levels. The HOMA-IR is increased in the diabetic control group due to increased serum blood glucose and decrease serum insulin level when compared to the normal control group. The OGTT is determined the efficacy of the body to tolerate 
the excess amount of glucose via insulin activity. In the diabetic control group, the FBS and HbAlc levels are increased and the serum insulin levels are decreased (Table 3). Treatment with Pioglitazone and E. parviflorum showed the ameliorative effect in diabetes by altering their levels in contrast to the diabetic control group. The Pioglitazone is a thiazolidinedione derivative which is a particular agonist for the nuclear peroxisome proliferator-activated receptor $\gamma(\operatorname{PPAR} \gamma)$. The PPAR $\gamma$ is found mainly in adipose tissues and in muscles. It upregulates the transcription and translocation of glucose transporter type 4 (GLUT-4) gene through which glucose entered into muscles and fats. The antihyperglycemic effect of pioglitazone is also due to suppression of hepatic gluconeogenesis, expression of genes for fatty acid (FFA) metabolism and lipid synthesis in adipose tissues. This mechanism of pioglitazone enhances insulin-sensitizing action. The therapeutic effect of E. parviflorum 400 might be due to its insulinotropic action.

The chronic elevation of fructose and dyslipidemia will deteriorate the antioxidant system of tissues by increased reactive oxygen species and lipid peroxidation ${ }^{[25,26]}$. Oxidative stress can damage mitochondria of pancreatic beta cells which lead to diabetes ${ }^{[27]}$. Pictomicrograph of the pancreatic islet (fig. 3) of disease control showed not only the derangement and necrotic cells but also decrease the numbers of $\beta$ cells.

These changes are prominently reflected in the decreased secretion of insulin level in the diabetic control group. Treatment with pioglitazone and E. parviflorum substantially protect the pancreas from destruction. This effect of E. parviflorum might be due to the presence of antioxidant principles like phenol, polyphenol groups in it which boost the antioxidant defence mechanism ${ }^{[11]}$. As the DNL process took place in the liver due to fructose administration, the liver enhances fatty acid deposition and subjected to produce more amount of SGOT, SGPT and ALP which collectively led to insulin resistance in the diabetic control group. The pioglitazone and E. parviflorum treated groups showed a hepatoprotective action by significantly decreasing those enzymes. The histopathological determination of the liver also refers to have hepatoprotective action of pioglitazone and E. parviflorum treated groups. Pictomicrograph of the liver is depicted in fig. 3(F-I), where the steatosis, necrosis and edema of connective tissues are visible in the diseased control groups. Treatment with pioglitazone and E. parviflorum maintain the architecture of hepatocyte near to normal control group.
This hepatoprotective effect of $E$. parviflorum is might be due to its anti-inflammatory action.

The TG, TC, HDL, VLDL and LDL levels determined at the end of the study. After the successful treatment of standard and test drugs for $6 \mathrm{w}$, we found that the TG, TC, VLDL and LDL levels of diabetic control groups are alleviated and HDL level is impaired due to received HFD-FRU-STZ induction when compared to the normal control group. The pioglitazone and E. parviflorum treated rats showed significantly $(\mathrm{p}<0.01)$ antihyperlipidemic action by altering those data of the diseased control group.

Pictomicrograph of adipose tissues is depicted in fig. 3(P-T) of the diabetic control group showed the large unilocular vacuole cells with respect to the presence of a large amount of storage lipid in it. Decreased size of vacuole cells reveals the ameliorative indications of pioglitazone and E. parviflorum in received HFDFRU-STZ induced type 2 diabetes when compared to the diseased control group.

In the present investigation, the inflammatory biomarkers like serum TNF $\alpha$ and hs-CRP are increased in the diseased control group when compared to the normal control groups. The TNF $\alpha$ is an inflammatory cytokine, released from mononuclear macrophage of adipose tissue. Chronic exposure to TNF $\alpha$ induces insulin resistance both in vitro and in vivo ${ }^{[28,29]}$. The insulin resistance is due to the inhibition of tyrosine kinase at the insulin receptor ${ }^{[30]}$. TNF $\alpha$ regulates the free fatty acid level in plasma and enhances lipid synthesis. Treatment with pioglitazone and E. parviflorum decrease the level of TNF $\alpha$ when compared to the diabetic control group. The significantly decreased level of TNF $\alpha$ in the E. parviflorum 400 group reveals is antihyperglycemic property improves insulin sensitivity ${ }^{[31,32]}$. C-reactive protein (CRP) is a $115 \mathrm{k}$ Da pentamer synthesized and released mainly by hepatocytes under the control of cytokines such as interleukin-6, interleukin-1, and TNF $\alpha$. CRP is a sensitive physiological marker of subclinical systemic inflammation that is associated with hyperglycemia, insulin resistance and overt Type 2 diabetes mellitus (T2DM). In the present investigation, the high sensitive C-reactive protein (hs-CRP) is increased in the diabetic control group due to received HFD-FRU-STZ. The pioglitazone and E. parviflorum showed anti-inflammatory action by significantly decreasing the serum hs-CRP when compared to the diabetic control group.

The excess consumption of dietary purine, highfructose corn syrup and table sugar produce more uric 
acid production. Hyperuricemia is occurred in obesity, diabetes (insulin resistance) and other condition such as hypertension, kidney disorder, reduced HDL cholesterol and hypertriglyceridemia ${ }^{[33,34]}$. Similarly, the elevated creatinine level refers to renal insufficiency during marked structural and functional abnormality of nephrons ${ }^{[35]}$. Hence, the serum uric acid level and creatinine level are estimated to support the ameliorative effect of pioglitazone and E. parviflorum in renal functions. This statement is further confirmed by the evidence of the histopathological study of the kidney (fig. 3[K-O]). The hyperlipidemia contributes to the development of diabetic nephropathy which can be treated with statins an antihyperlipidemic drug $^{[36]}$. Diabetic nephropathy involved in uncontrolled hyperglycemia and the glucose level is an important predictor of terminal kidney failure ${ }^{[37]}$. The diabetic nephropathy involved in various changes in kidney like glomerular hypertrophy, hyperfiltration, tubular atrophy, increased mesangial cell number and mesangial matrix, increased thickness of basement membranes of glomerulus and tubules ${ }^{[38]}$. The aldol reductase (AR) enzyme has active participation in the development of diabetic nephropathy through polyol pathway, production of dicarbonyl molecules and by producing reactive oxygen species in the kidney ${ }^{[39]}$. Similarly, the oral hypoglycemic drug pioglitazone was shown to reduce fibrosis, mesangial proliferation and inflammation ${ }^{[40]}$ in diabetic nephropathy. In the present investigation, the E. parviflorum treated groups maintained the architecture of renal tissues like normal control group might be due to inhibiting the aldol reductase enzyme ${ }^{[7]}$. The healing effect of $E$. parviflorum in diabetic nephropathy might be due to its Cyclooxygenase-2 (COX-2) inhibitory action which is similar report of previous researchers ${ }^{[41,42]}$.

\section{CONCLUSION}

In conclusion, the HFD-FRU-STZ can be used together to induce a diabetic model in animals which can evoke similar co-morbidity conditions as in type 2 diabetic patients through various cellular mechanisms. We believe our phenotype model can meet the standard in low expensive as compared to others and genotype models. The oral administration of E. parviflorum extract has ameliorated the abnormal consequences of diabetes by improving antioxidant effect, insulinotropic effect, glucose metabolism, fat metabolism, liver functions and histopathological architecture to some extent.

\section{Sources of funding:}

University Grant Commission New Delhi (Junior Research Fellowship).

\section{Acknowledgments:}

The author expresses the sincere obligation towards Andhra University college of Pharmaceutical Sciences, Visakhapatnam, Andhra Pradesh for their support in the accomplishment of research work.

\section{Conflict of interest:}

All authors report no conflicts of interest in this work.

\section{REFERENCES}

1. Zhang M, Lv XY, Li J, Xu ZG, Chen L. The characterization of high-fat diet and multiple low-dose streptozotocin induced type 2 diabetes rat model. Exp Diabetes Res 2008;2008:1-9.

2. Bansal P, Paul P, Mudgal J, Nayak PG, Pannakal ST, Priyadarsini $\mathrm{KI}$, et al. Antidiabetic, antihyperlipidemic and antioxidant effects of the flavonoid rich fraction of Pilea microphylla (L.) in high fat diet/streptozotocin-induced diabetes in mice. Exp Toxicol Pathol 2012;64(6):651-8.

3. Gothandam K, Ganesan VS, Ayyasamy T, Ramalingam S. Antioxidant potential of theaflavin ameliorates the activities of key enzymes of glucose metabolism in high fat diet and streptozotocin-induced diabetic rats. Redox Rep 2019;24(1):41-50.

4. Bamosa A, Elnour A, Kaatabi H, Al Meheithif A, Aleissa K, Al-Almaie S. Zamzam water ameliorates oxidative stress and reduces hemoglobina1c in type 2 diabetic patients. J Diabetes Metab 2013;4(249):2.

5. Prabhakar PK, Doble M. Interaction of phytochemicals with hypoglycemic drugs on glucose uptake in L6 myotubes. Phytomedicine 2011;18(4):285-91.

6. Augustine AW, Narasimhan A, Vishwanathan M, Karundevi B. Evaluation of antidiabetic property of Andrographis paniculata powder in high fat and sucrose-induced type-2 diabetic adult male rat. Asian Pac J Trop Dis 2014;4:S140-7.

7. Schepetkin IA, Ramstead AG, Kirpotina LN, Voyich JM, Jutila MA, Quinn MT. Therapeutic potential of polyphenols from Epilobium angustifolium (Fireweed). Phytother Res 2016;30(8):1287-97.

8. Kiss AK, Bazylko A, Filipek A, Granica S, Jaszewska E, Kiarszys U, et al. Oenothein B's contribution to the antiinflammatory and antioxidant activity of Epilobium sp. Phytomedicine 2011;18(7):557-60.

9. Ducrey B, Marston A, Göhring S, Hartmann RW, Hostettmann K. Inhibition of $5 \alpha$-reductase and aromatase by the ellagitannins oenothein A and oenothein B from Epilobium species. Planta Med 1997;63(02):111-4.

10. Steenkamp V, Gouws MC, Gulumian M, Elgorashi EE, Van Staden J. Studies on antibacterial, anti-inflammatory and antioxidant activity of herbal remedies used in the treatment of benign prostatic hyperplasia and prostatitis. J Ethnopharmacol 2006;103(1):71-5.

11. Hevesi BT, Houghton PJ, Habtemariam S, Kéry Á. Antioxidant and antiinflammatory effect of Epilobium parviflorum Schreb. Phytother Res 2009;23(5):719-24.

12. Wu D, Wen W, Qi CL, Zhao RX, Lü JH, Zhong CY, et al. 
Ameliorative effect of berberine on renal damage in rats with diabetes induced by high-fat diet and streptozotocin. Phytomedicine 2012;19(8-9):712-8.

13. Muniyappa R, Lee S, Chen H, Quon MJ. Current approaches for assessing insulin sensitivity and resistance in vivo: advantages, limitations and appropriate usage. Am J Physiol Endocrinol Metab 2008;294(1):E15-26.

14. Anitha M, Sakthidevi G, Muthukumarasamy S, Mohan VR. Effect of Cynoglossum zeylanicum (Vehl ex Hornem) Thunb. ex Lehm on oral glucose tolerance in rats. J Appl Pharm Sci 2012;2(11):75-8.

15. Srinivasan K, Patole PS, Kaul CL, Ramarao P. Reversal of glucose intolerance by pioglitazone in high fat diet-fed rats. Methods Find Exp Clin Pharmacol 2004;26(5):327-3.

16. Patel J, Iyer A, Brown L. Evaluation of the chronic complications of diabetes in a high fructose diet in rats. Indian J Biochem Bio 2009:46:66-72.

17. Veerapur VP, Prabhakar KR, Thippeswamy BS, Bansal P, Srinivasan KK, Unnikrishnan MK. Antidiabetic effect of Dodonaea viscose (L). Lacq. Aerial parts in high fructose-fed insulin resistant rats: A mechanism based study. Indian J Exp Biol 2010;48:800-10.

18. Wilson RD, Islam MS. Fructose-fed streptozotocin-injected rat: an alternative model for type 2 diabetes. Pharmacol Rep 2012;64(1):129-39.

19. Bigoniya P, Nishad R, Singh CS. Preventive effect of sesame seed cake on hyperglycemia and obesity against high fructose-diet induced Type 2 diabetes in rats. Food Chem 2012;133(4):1355-61.

20. Sanders FW, Griffin JL. De novo lipogenesis in the liver in health and disease: more than just a shunting yard for glucose. Biol Rev 2016;91(2):452-68.

21. Reyna SM, Ghosh S, Tantiwong P, Meka CR, Eagan P, Jenkinson CP, et al. Elevated toll-like receptor 4 expression and signaling in muscle from insulin-resistant subjects. Diabetes 2008;57(10):2595-602.

22. Deutschländer MS, Lall N, Van de Venter M, Dewanjee S. The hypoglycemic activity of Euclea undulata Thunb. var. myrtina (Ebenaceae) root bark evaluated in a streptozotocinnicotinamide induced type 2 diabetes rat model. S Afr J Bot 2012;80:9-12.

23. Lukic ML, Stošic-Grujicic S, Shahin A. Effector mechanisms in low-dose streptozotocin-induced diabetes. Dev Immunol 1998;6(1-2):119-28.

24. Soling HD, Kleineke J. Species dependent regulation of hepatic gluconeogenesis in higher animals. In: Hanson RW, Mehlman MA, editors. Gluconeogenesis: its regulation in mammalian species. New York: Wiley Intersci 1976:369-462.

25. Galipeau D, Verma S, McNeill JH. Female rats are protected against fructose-induced changes in metabolism and blood pressure. Am J Physiol Heart Circ Physiol 2002;283(6):H247884.

26. Yadav H, Jain S, Sinha PR. Antidiabetic effect of probiotic dahi containing Lactobacillus acidophilus and Lactobacillus casei in high fructose fed rats. Nutrition 2007;23(1):62-8.

27. Jamieson D, Chance B, Cadenas E, Boveris A. The relation of free radical production to hyperoxia. Annu Rev Physiol 1986;48(1):703-19.

28. Navarro JF, Mora C. Role of inflammation in diabetic complications. Nephrol Dial Transplant 2005;20:2601-4.

29. Swaroop JJ, Rajarajeswari D, Naidu JN. Association of TNF- $\alpha$ with insulin resistance in type 2 diabetes mellitus. Indian $\mathrm{J}$ Med Res 2012;135(1):127.

30. Chen $\mathrm{P}$, Zhang Q, Dang $\mathrm{H}$, Liu X, Tian $\mathrm{F}$, Zhao J, et al. Antidiabetic effect of Lactobacillus casei CCFM0412 on mice with type 2 diabetes induced by a high-fat diet and streptozotocin. Nutrition 2014;30(9):1061-8.

31. Hotamisligil GS, Shargill NS, Spiegelman BM. Adipose expression of tumor necrosis factor-alpha: direct role in obesity-linked insulin resistance. Science 1993;259(5091):8791.

32. Cheung AT, Ree D, Kolls JK, Fuselier J, Coy DH, Bryer-Ash $\mathrm{M}$. An in vivo model for elucidation of the mechanism of tumor necrosis factor- $\alpha(\mathrm{TNF}-\alpha)$-induced insulin resistance: evidence for differential regulation of insulin signaling by TNF- $\alpha$. Endocrinology 1998;139(12):4928-35.

33. Angelopoulos TJ, Lowndes J, Zukley L, Melanson KJ, Nguyen V, Huffman A, et al. The effect of high-fructose corn syrup consumption on triglycerides and uric acid. J Nutr 2009;139(6):1242S-5S.

34. Onat A, Uyarel H, Hergenç G, Karabulut A, Albayrak S, Sarı I, et al. Serum uric acid is a determinant of metabolic syndrome in a population-based study. Am J Hypertens 2006;19(10):105562.

35. Patschan D, Müller GA. Acute kidney injury in diabetes mellitus. Int J Nephrol 2016;2016:1-8.

36. Tonolo G, Velussi M, Brocco E, Abaterusso C, Carraro A, Morgia G, et al. Simvastatin maintains steady patterns of GFR and improves AER and expression of slit diaphragm proteins in type II diabetes. Kidney Int 2006;70(1):177-86.

37. Bash LD, Selvin E, Steffes M, Coresh J, Astor BC. Poor glycemic control in diabetes and the risk of incident chronic kidney disease even in the absence of albuminuria and retinopathy: Atherosclerosis Risk in Communities (ARIC) Study. Arch Intern Med 2008;168(22):2440-7.

38. Vujicic B, Turk T, Crncevic-Orlic Z, Dordevic G, Racki S. Pathophysiology and Complications of Diabetes Mellitus. Diabetic Nephropathy In Tech 2012;71-96.

39. Dunlop M. Aldose reductase and the role of the polyol pathway in diabetic nephropathy. Kidney Int 2000;77:3-12.

40. Weissgarten J, Berman S, Efrati S, Rapaport M, Averbukh Z, Feldman L. Apoptosis and proliferation of cultured mesangial cells isolated from kidneys of rosiglitazone-treated pregnant diabetic rats. Nephrol Dial Transplant 2006;21(5):1198-204.

41. Wang JL, Cheng HF, Shappell S, Harris RC. A selective cyclooxygenase- 2 inhibitor decreases proteinuria and retards progressive renal injury in rats. Kidney Int 2000;57(6):233442.

42. Yao B, Xu J, Qi Z, Harris RC, Zhang MZ. Role of renal cortical cyclooxygenase-2 expression in hyperfiltration in rats with high-protein intake. Am J Physiol Renal Physiol 2006;291(2):F368-74. 